\title{
Transcutaneous auricular vagus nerve stimulation holds potential to suppress COVID-19 cytokine storm
}

\author{
Patrick Nemechek* \\ Nemechek Autonomic Medicine, 4252 N Verrado Way Suite 200, Buckeye, AZ 85396 Buckeye, USA
}

\begin{abstract}
Coronavirus disease 2019 (COVID-19) continues to exert mortality worldwide largely due to a lack of treatment options to prevent or ameliorate advanced respiratory dysfunction. Knowledge of the COVID-19 virus-host interaction is rapidly evolving, and significant evidence indicates that an uncontrolled immune response resulting in a cytokine storm can trigger rapid decline. Secondary to massive efforts to slow the spread of the novel coronavirus responsible for COVID-19, antiviral agents and inflammation suppression minimizing cytokine storm development most likely will be required to improve survival rates in hospitalized COVID-19 patients. Vagus nerve stimulation (VNS) is safe and several lines of evidence suggest it is potent enough to regulate the high levels of pro-inflammatory cytokines seen in hospitalized COVID-19 patients. Based on its success in mitigating both chronic and acute inflammation without triggering harmful immunosuppression, transcutaneous auricular vagus nerve stimulation (taVNS) presents a novel potential treatment for COVID-19. A clinical trial is being designed to determine whether taVNS can significantly improve the mortality rate among hospitalized COVID-19 patients by preventing a fatal immune response.
\end{abstract}

\section{Introduction}

Coronavirus disease 2019 (COVID-19) has a high mortality rate among hospitalized patients and presents an unprecedented challenge for healthcare systems. In China, where the virus originated, 4,637 COVID-19 deaths have been reported while deaths in the U.S., Italy, and the U.K. have soared to $64,943,28,236$, and 27,583 , respectively, as of May 1, 2020 [1].

Many hospitalized COVID-19 patients develop acute respiratory distress syndrome (ARDS) [2] and have high inflammatory markers suggestive of a cytokine storm (Figure 1). Serum cytokines measured in some of the first Chinese COVID-19 patients to be evaluated were significantly higher in severe compared to moderate cases (Figure 2), specifically the pro-inflammatory cytokines interleukin $2 \mathrm{R}$ (IL-2R), IL6 , IL-10, and tumor necrosis factor $\alpha$ (TNF- $\alpha$ ) [3]. The cytokine storm typically leads to immune failure, organ failure, secondary infection, and ultimately death $[3,4]$.

The factors precipitating cytokine storm in a subset of patients remain unclear. COVID-19 patients receive supportive measures such as fluids, antibiotics for secondary infections, mechanical ventilation, and pressure support, but in the presence of cytokine storm, outcomes are poor despite such measures [5] (Figure 3).

Risk factors for severe COVID-19 include chronic conditions associated with excessive inflammation such as respiratory and cardiovascular diseases, cancer, hypertension, diabetes, obesity, smoking, and old age [6] (Figure 4). Regardless of COVID-19 infection, older individuals generally experience inflammaging, which is the presence of chronic, low-grade inflammation [7]. With a higher baseline of pre-existing inflammation compared to younger patients, patients over age 60 are more likely to develop an uncontrolled immune response and the dangerous cytokine storm if they contract the virus.

The relationship between COVID-19 severity and chronic inflammatory conditions suggests that a preexisting, heavy inflammatory load is the common thread among those most at risk (Figure 5).
Dual therapy aimed at reducing the infectious process and improving cytokine regulation is needed to improve COVID-19 outcomes. In addition to supportive care, antiviral medication should be used to block viral replication in conjunction with anti-inflammatory therapy to improve immune regulation. Steroids, anti-inflammatory and anti-cytokine drugs such as tocilizumab, and anti-cytokine signaling drugs such as janus kinase inhibitors are proposed to help regulate inflammation and viral entry in COVID-19 [8]. These drugs are costly, however, and often result in increased risk of secondary infection due to general suppression of the immune system $[9,10]$.

The poor regulation of immune response observed in cytokine storm COVID-19 patients may result from the inability of the vagus nerve to properly regulate the immune system. The vagus nerve is involved in an inflammation controlling reflex triggered when the afferent vagus nerve senses inflammatory products through peripheral receptors (Figure 6). Vagus nerve activity is relayed through the central nervous system to the efferent vagus nerve. This pathway involves the splenic nerve, which when activated releases norepinephrine and results in suppression of proinflammatory cytokine production by macrophages and alleviates inflammation in many pathological settings (e.g., endotoxemia, peritonitis, or acute kidney injury) [11].

Electrical stimulation of the vagus nerve using vagus nerve stimulation (VNS) can improve the body's natural ability to regulate the inflammatory response and may be potent enough to suppress proinflammatory cytokines and prevent death from COVID-19, especially if used early enough in the course of hospitalization [12-17].

${ }^{*}$ Correspondence to: Patrick Nemechek, Nemechek Autonomic Medicine, 4252 N Verrado Way Suite 200, Buckeye, AZ 85396 Buckeye, USA, E-mail: dr@ autonomicmed.com

Received: May 15, 2020; Accepted: June 05, 2020; Published: June 10, 2020 


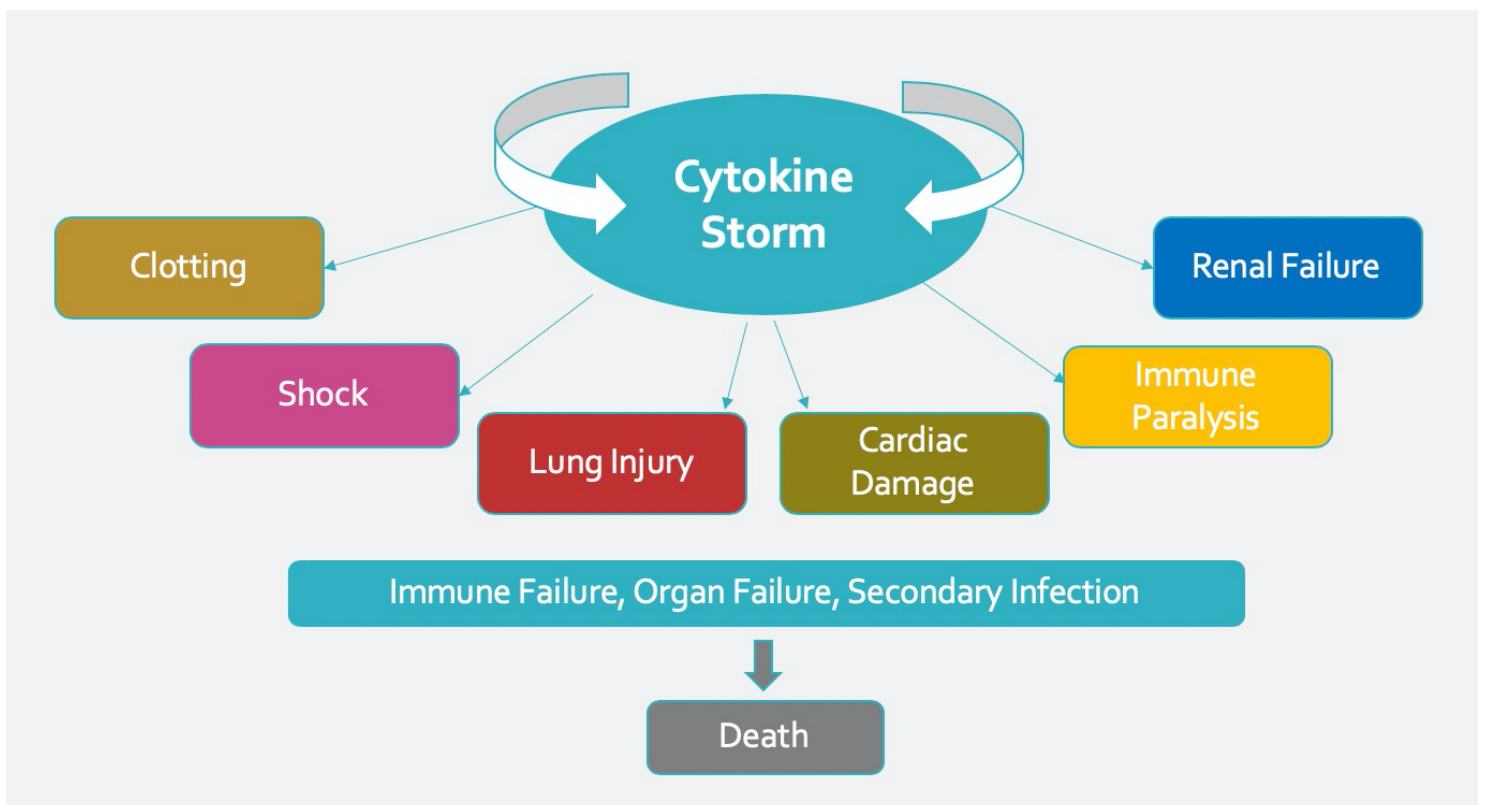

Figure 1. Cytokine storm induced by COVID-19 infection in some patients results in organ damage followed by immune failure, organ failure, secondary infection, and death.

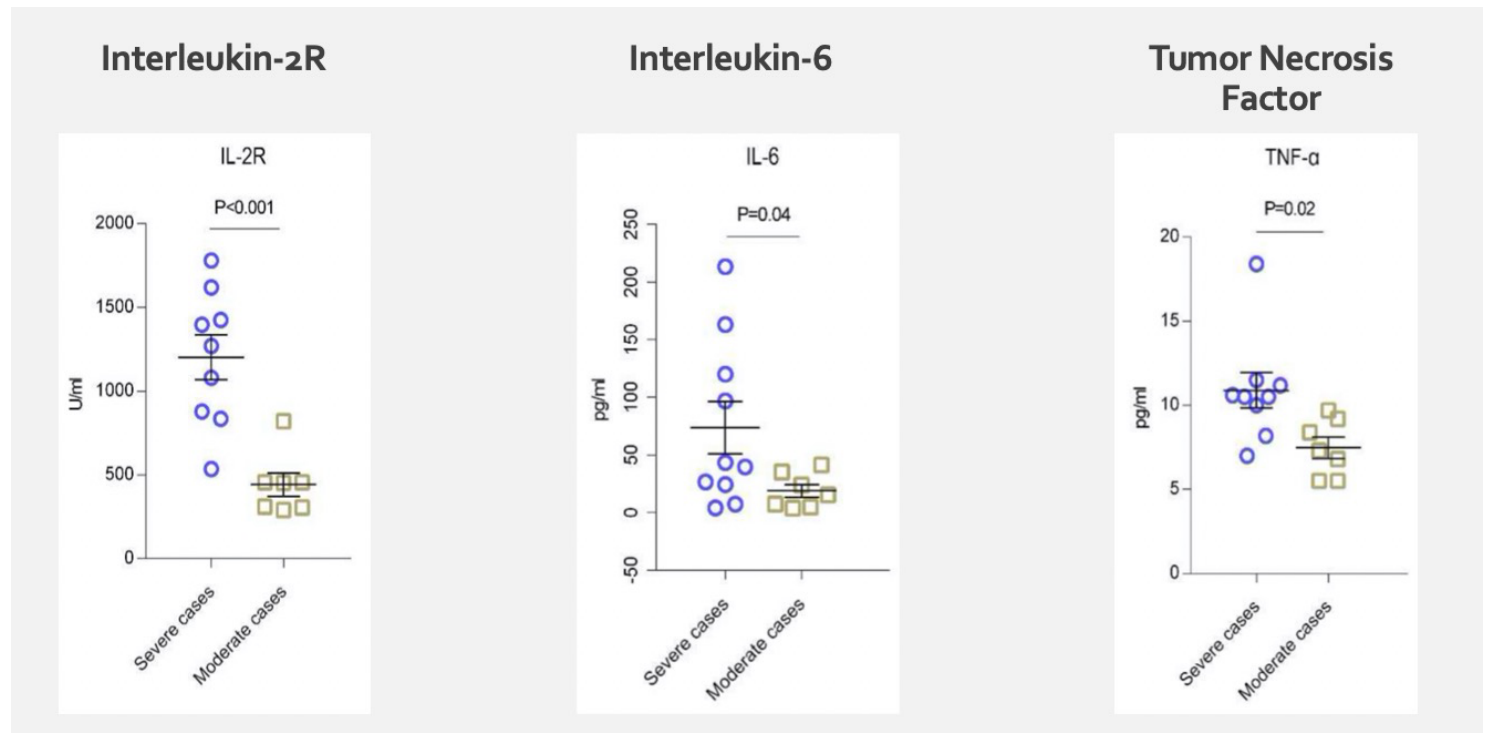

Figure 2. Elevated serum cytokines observed in severe COVID-19 cases. From Chen G et al., Clinical and immunologic features in severe and moderate Coronavirus Disease [3].

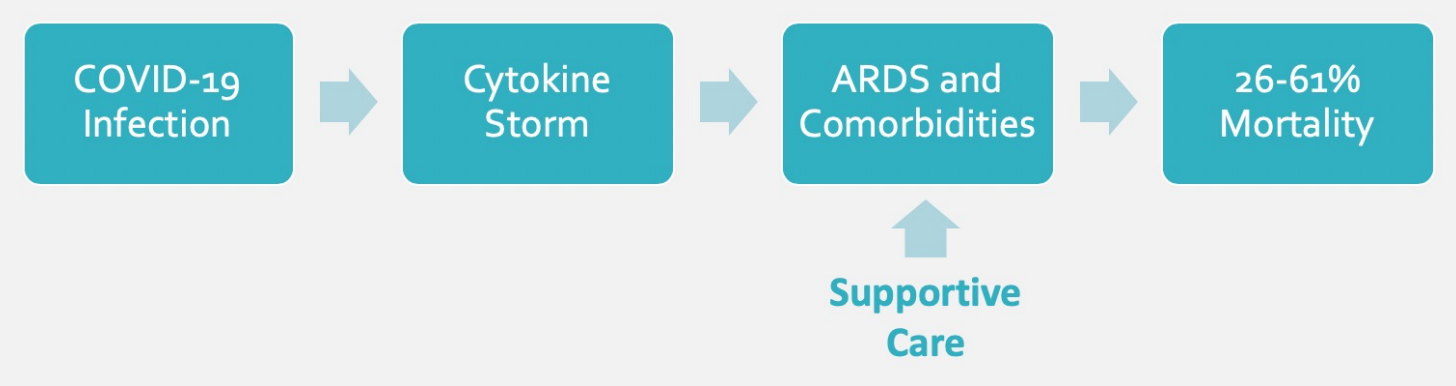

Figure 3. Typical progression of clinical presentation following COVID-19 infection. Following the onset of cytokine storm, patients often experience ARDS and have a high mortality rate despite supportive care measures. 


\section{COVID-19 Risk Factors}

- COPD

- CAD

- Obesity (BMl>40)

- Diabetes Mellitus

- Chronic Kidney Disease

- Older Patients (>6o yr.)

\section{Elevated Pro-Inflammatory State}

- COPD

- CAD

- Obesity (BMI>40)

- Diabetes Mellitus

- Chronic Kidney Disease

- Older Patients (>6o yr.)

- Chronic Metabolic Inflammation (Inflammaging)

Figure 4. Risk factors for severe reaction to COVID-19 and elevated pro-inflammatory state include conditions that already involve high levels of inflammation.

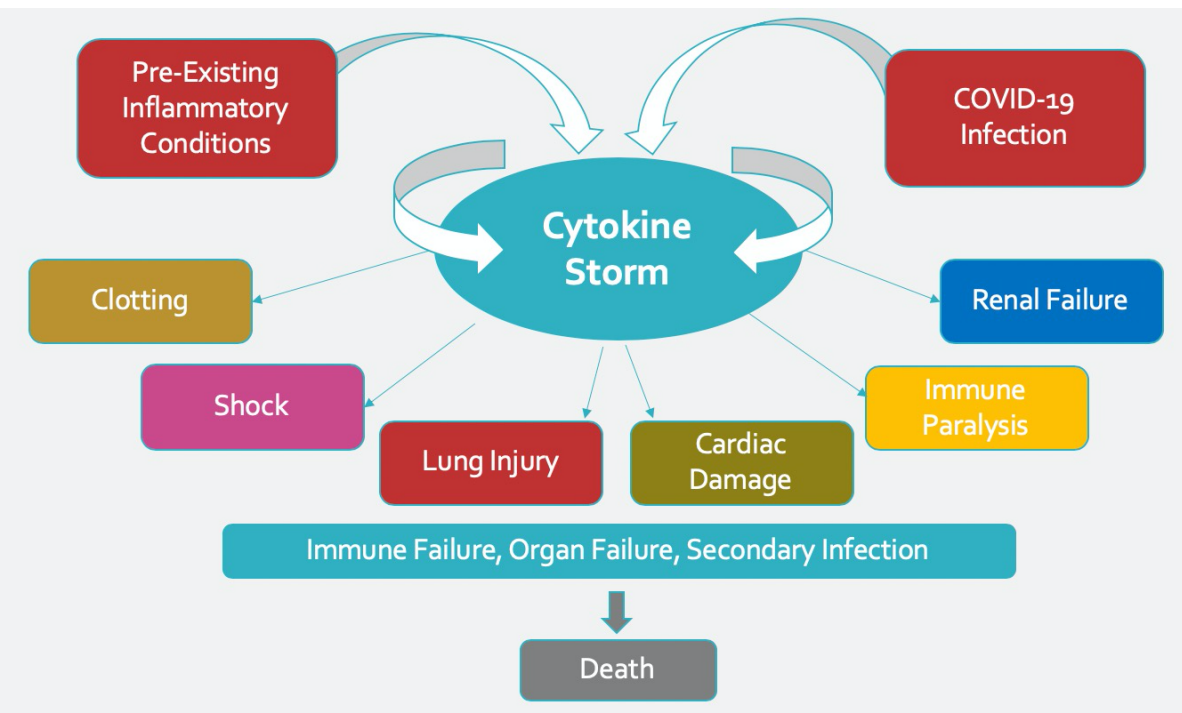

Figure 5. Pre-existing inflammatory conditions are characterized by a baseline elevated inflammatory load made even larger upon COVID-19 infection.

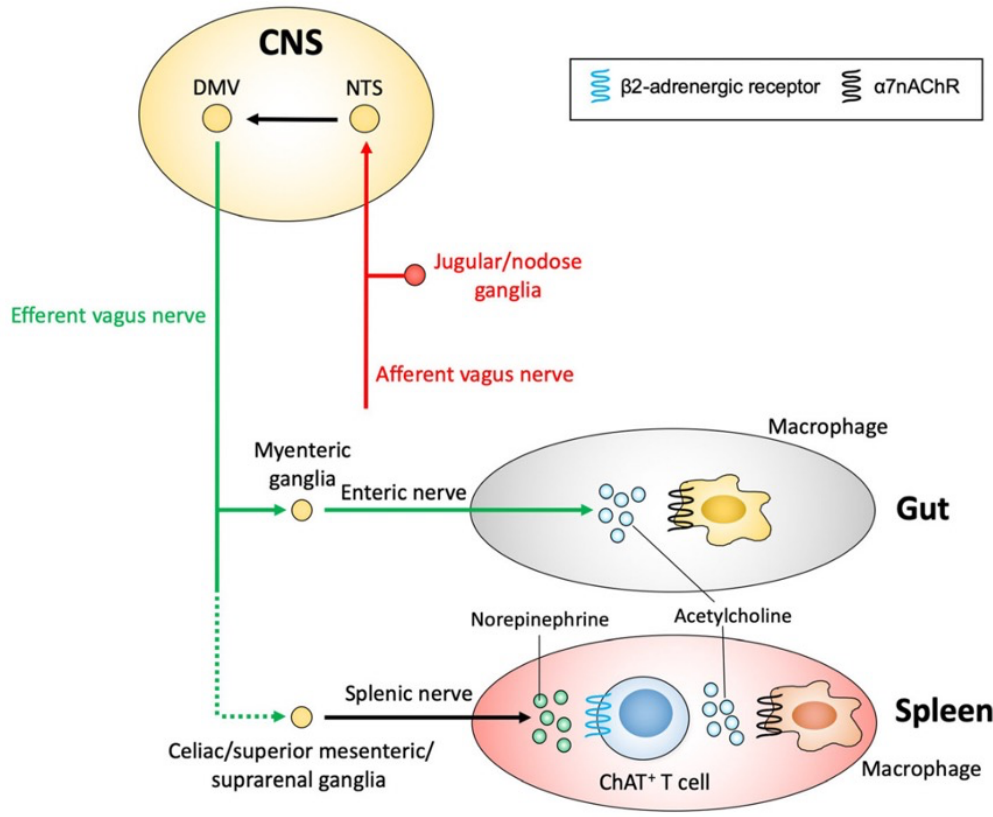

Figure 6. The vagus inflammatory reflex [11]. 
In rat models of sepsis, VNS attenuates the release of proinflammatory cytokines, prevents hypotension, modulates coagulation, and prevents fibrinolysis activation, decreasing organ dysfunction and improving survival [18]. Human studies also demonstrate that VNS suppresses the production of proinflammatory cytokines and improves clinical symptoms in rheumatoid arthritis, intractable epilepsy, atrial fibrillation, and Crohn's Disease [19-22]. This suggests that VNS may be effective in treating disorders characterized by cytokine dysregulation and that it has the potential to prevent hospitalized patients with COVID-19 from progressing to respiratory failure and death.

The vagus nerve can be electrically stimulated with an implantable device placed on the vagus nerve or by stimulating the auricular branch of the vagus nerve with a conductive surface in contact with the concha of the ear (transcutaneous auricular VNS; taVNS). Vagus nerve stimulation via afferent vagus nerve fibers in the ear activates the inflammatory reflex and regulates the immune response [23]. taVNS significantly reduces systemic pro-inflammatory cytokines [15] and improves clinical outcome in a variety of medical conditions involving uncontrolled immune activation in instances of chronic inflammation, such as rheumatoid arthritis, and acute inflammation, such as sepsis $[13,24]$. Vagus nerve stimulation is safe and effective in regulating inflammatory cytokines without suppressing the immune system [25]. Indeed, an implantable VNS device successfully reduced serum cytokines TNF, IL-1 $\beta$, and IL-6 in epilepsy patients [19] suggesting that VNS can therapeutically assist the vagus nerve in modulating cytokine production (Figure 7).

taVNS is applied on the outer ear using a simple device that requires minimal training and is inexpensive, readily manufactured, and reusable, making it an ideal therapeutic option in a global pandemic affecting many resource-limited countries. Based on its success in modulating immune reactions, taVNS may prevent and even counteract the cytokine storm observed among some hospitalized COVID-19 patients (Figure 8).

A dual approach to COVID-19 leveraging the blockage of viral replication along with taVNS may help to prevent ARDS, the deadly cytokine storm and system failure, and significantly improve survival. Indeed, a recent case report described clinical improvement in the form of improved respiratory capacity in two COVID-19 patients treated with non-invasive VNS [26].

\section{Proposed study}

The proposed interventional, non-randomized, non-controlled, single-arm open label prospective study will determine the therapeutic effect and tolerance of taVNS in patients with moderate, severe, or critical ARDS associated with COVID-19. A single arm was chosen for this pilot study to assess the efficacy and tolerability of novel regimens before proceeding to a larger randomized controlled trial.

In addition to standard care, five minutes of taVNS will be delivered four times daily (every six hours) to volunteer adult patients hospitalized with COVID-19. This dosing is based on previous reports that increased dosing produces improved clinical outcome [19]. Patients who choose not to receive taVNS will receive standard of care alone. Standard care includes the use of anti-viral and antiinflammatory agents as deemed necessary by the treating physician.

Electrical stimulation will be applied with smart-phone based device that has been approved by the Taiwanese FDA (TFDFA) to function as a TENS (transcutaneous electrical nerve stimulation) unit, and is presently sold in the U.S. as a consumer wellness product (The Vitality Smartcable).
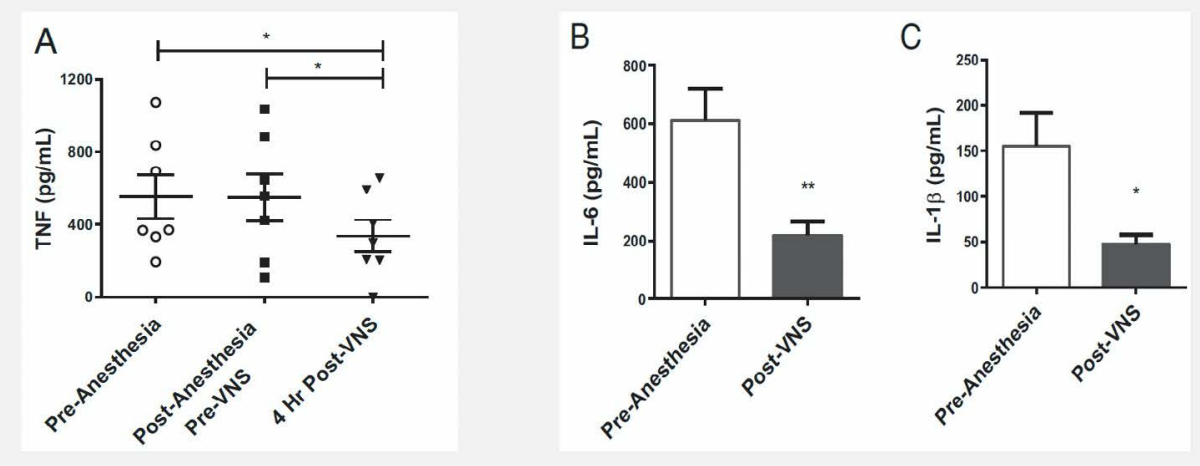

Figure 7. VNS significantly reduced TNF, IL-1 $\beta$, and IL-6 cytokines in epilepsy patients. From Koopman et al., Vagus nerve stimulation inhibits cytokine production and attenuates disease severity in rheumatoid arthritis [19].

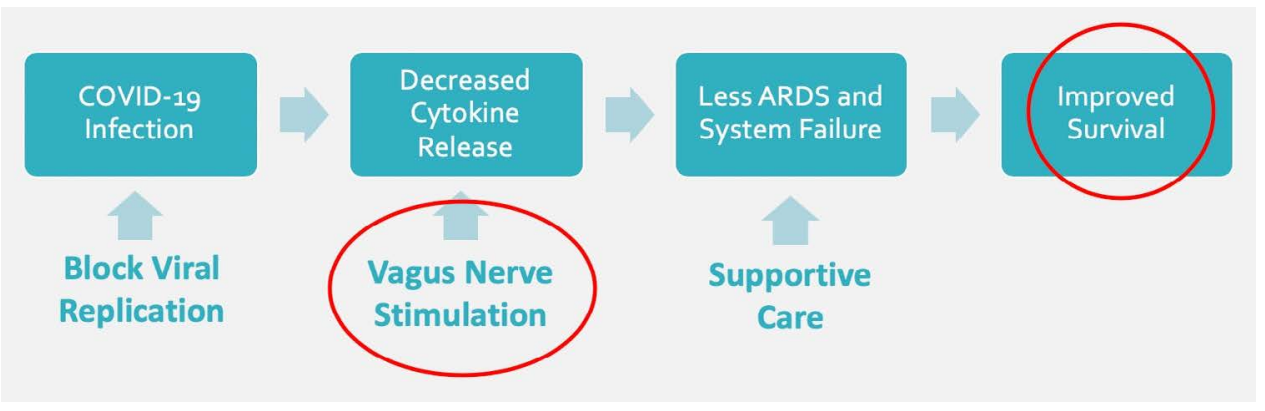

Figure 8. The addition of VNS to COVID-19 patient care may decease cytokine release and avoid ARDS and cytokine storm resulting in improved survival. 
The Vitality Smartcable is not approved as a medical device by the U.S. FDA but an Emergency Use Application requesting its use has been submitted to the FDA. TENS units are considered Class 1 medical devices and are safe enough that they are sold over the counter without a physician's prescription in the U.S.

The Vitality Smartcable uses a specialized ear clip with one contact placed within the concha and the other contact of the clip acting as the grounding element on the back of the ear. Stimulation will be set at 5 $\mathrm{Hz}, 800 \mathrm{mS}$ with voltage set at $10 \mathrm{~V}$ below the perceptual threshold.

\section{Eligibility criteria}

Male and female patients with COVID-19 infection confirmed via any readily available test, requiring hospital admission, and over 60 years of age will be recruited for participation in the study. All patients must present with fever and respiratory distress ( $\geq 30$ breaths/ min, oxygen saturation $\leq 93 \%$ at rest in ambient air, or oxygen saturation $\leq 97 \%$ with $\mathrm{O}_{2}>5 \mathrm{~L} / \mathrm{min}, \mathrm{PaO}_{2} / \mathrm{FiO}_{2} \leq 300 \mathrm{mmHg}$ ) with radiological findings of pneumonia.

Exclusion criteria include known intolerance to vagus nerve stimulation, pregnancy, potentially life-threatening heart arrhythmia, symptomatic bradycardia, and current documented bacterial infection. Abnormal laboratory results requiring medication may be discussed on a case-by-case basis.

\section{Outcome Measures}

The primary outcome measure will be survival without need of ventilator at day 14 after treatment initiation. This includes both nonintubated patients and cumulative incidence of successful tracheal extubation at day 14. Patients in need of ventilator at day 14 (including non-invasive ventilation) or who have died will be considered a negative outcome. New do-not-resuscitate orders will be considered a negative outcome.

The World Health Organization (WHO) progression scale (see below) will be used to score patients based on symptomology and respiratory support requirements at days 4,7 , and 14 of hospitalization. Additional metrics will comprise overall survival, ventilator-free days, respiratory acidosis at day 4 , time to oxygen supply independence, duration of hospitalization, time to intensive care discharge, and time to hospital discharge.

\section{WHO progression scale:}

- 0 - Uninfected; no viral RNA detected

- 1 - Asymptomatic; viral RNA detected

- 2 - Symptomatic; independent

- 3 - Symptomatic; assistance needed

- 4 - Hospitalized; no oxygen therapy

- 5 - Hospitalized; oxygen by mask or nasal prongs

- 6 - Hospitalized; oxygen by NIV or high flow

- 7 - Intubation and mechanical ventilation, $\mathrm{pO} 2 / \mathrm{FIO} 2>/=150$ or $\mathrm{SpO} 2 / \mathrm{FIO} 2>/=200$

- 8 - Mechanical ventilation, (pO2/FIO2 $<150$ or SpO2/FIO2 <200) or vasopressors (norepinephrine $>0.3 \mathrm{microg} / \mathrm{kg} / \mathrm{min}$ )

- 9 - Mechanical ventilation, pO2/FIO2 < 150 and vasopressors (norepinephrine $>0.3 \mathrm{microg} / \mathrm{kg} / \mathrm{min}$ ), or dialysis, or ECMO

- 10 - Dead
Based on Simon's two-stage minimax analysis using a significance level of 0.05 and $80 \%$ power, at least 13 patients will need to be recruited to determine a clinical benefit rate of at least $50 \%$. If none of the first six patients to be treated with taVNS are free of ventilator support after using taVNS for 14 days, the study will be stopped. If three out of 13 patients are free of ventilator support, the treatment will be assumed to be successful and further investigation will be warranted.

\section{Summary}

Many of the proposed pharmaceutical treatments for COVID-19 modulate the inflammatory response but may also suppress the immune system and allow for opportunistic secondary infection. Vagus nerve stimulation is safe and effective in suppressing excessive immune response without the risk of immune suppression. taVNS is a promising technology for simple, non-invasive, inexpensive, and reusable vagus nerve stimulation.

As adjunctive therapy, taVNS has a proven track record for safety and is not expected to interfere with other treatment regimens. If successful in improving outcomes of COVID-19, taVNS devices can be made rapidly and inexpensively and can be reused after sterilization since they simply clip on to a patient's outer ear. This strategy represents an ideal countermeasure for healthcare systems struggling to successfully treat COVID-19 patients. Institutional review board approval from Western IRB is currently being sought for this study and clinical partners are needed to assist with its implementation.

\section{Competing interest statement}

Dr. Nemechek is co-founder of Nemechek Technologies and received renumeration from Nemechek Technologies.

Funding statement: There was no external funding support for this manuscript.

Author declarations: Patrick Nemechek is co-founder and CEO of Nemechek Technologies, a company which sells a consumer wellness electric neuromodulator. All relevant ethical guidelines have been followed; any necessary IRB and/or ethics committee approvals have been obtained and details of the IRB/oversight body are included in the manuscript. All necessary patient/participant consent has been obtained and the appropriate institutional forms have been archived.

I understand that all clinical trials and any other prospective interventional studies must be registered with an ICMJE-approved registry, such as ClinicalTrials.gov. I confirm that any such study reported in the manuscript has been registered and the trial registration ID is provided (note: if posting a prospective study registered retrospectively, please provide a statement in the trial ID field explaining why the study was not registered in advance).

\section{References}

1. Coronavirus COVID-19 global cases by the Center for Systems Science and Engineering (CSSE) at Johns Hopkins University (JHU). Updated May 1, 2020. Accessed May 2. 2020. Available at: https://coronavirus.jhu.edu/map.html.

2. Xu Z, Shi L, Wang Y, Zhang J, Huang L, et al. (2020) Pathological findings of COVID-19 associated with acute respiratory distress syndrome. Lancet Respir Med 2020: 420-422. [Crossref]

3. Chen G, Wu D, Guo W, Cao Y, Huang D, et al. (2020) Clinical and immunologic features in severe and moderate Coronavirus Disease 2019. J Clin Invest pii: 137244. [Crossref]

4. Huang C, Wang Y, Li X, Ren L, Zhao J, Hu Y, et al. (2020) Clinical features of patients infected with 2019 novel coronavirus in Wuhan, China. Lancet 395: 497-506. [Crossref] 
5. Yang Y, Shen C, Li J, Yuan J, Yang M, et al. (2020) Exuberant elevation of IP-10, $\mathrm{MCP}-3$, and Il-1ra during SARS-CoV-2 infection is associated with disease severity and fatal outcome. medRxiv 20029975.

6. Jordan RE, Adab P, Cheng KK (2020) Covid-19 risk factors for severe disease and death. BMJ 368: m1198. [Crossref]

7. Franceschi C, Garagnani P, Parini P, Giuliani C, Santoro A (2018) Inflammaging: a new immune-metabolic viewpoint for age-related diseases. Nature Reviews Endocrinology 14: 576-590. [Crossref]

8. Mehta P, McAuley DF, Brown M, Sanchez E, Tattersall RS, et al. (2020) COVID-19: consider cytokine storm syndromes and immunosuppression. Lancet 395: 1033-1034. [Crossref]

9. Ali T, Kaitha S, Mahmood S, Ftesi A, Stone J, Bronze M (2013) Clinical use of antiTNF therapy and increased risk of infections. Drug Healthc Patient Saf 5: 79-99. [Crossref]

10. Lang V, Englbrecht M, Rech J, Nusslein H, Manger K, Schuch F, et al. (2012) Risk of infections in rheumatoid arthritis patients treated with tocilizumab. Rheumatol 5: 852-857. [Crossref]

11. Tanaka S, Hammond B, Rosin DL, Okusa MD (2019) Neuroimmunomodulation of tissue injury and disease: an expanding view of the inflammatory reflex pathway. Bioelectron Med 5: 13. [Crossref]

12. Kaniusas E, Kampusch S, Tittgemeyer M (2019) Current Directions in the Auricular Vagus Nerve Stimulation I - A Physiological Perspective. Front Neurosci 13: 854. [Crossref]

13. Koopman FA (2016)) Vagus nerve stimulation inhibits cytokine production and attenuates disease severity in rheumatoid arthritis. Proc Natl Acad Sci U S A 113: 8284-8289. [Crossref]

14. de Jonge WJ, van der Zanden EP, The FO (2005) Stimulation of the vagus nerve attenuates macrophage activation by activating the Jak2-STAT3 signaling pathway. Nat Immunol 6: 844-851. [Crossref]

15. Zhao YX, He W, Jing XH, Liu JL, Rong PJ, et al. (2012) Transcutaneous auricular vagus nerve stimulation protects endotoxemic rat from lipopolysaccharide-induced inflammation. Evid Based Complement Alternat Med 2012: 627023. [Crossref]
16. Stavrakis S, Humphrey MB, Scherlag B (2017) Low-Level Vagus Nerve Stimulation Suppresses Post-Operative Atrial Fibrillation and Inflammation: A Randomized Study. JACC Clin Electrophysiol 3: 929-938. [Crossref]

17. Yamakawa K, Matsumoto N, Imamura Y, Muroya T, Yamada T, et al. (2013) Electrica vagus nerve stimulation attenuates systemic inflammation and improves survival in a rat heatstroke model. PloS One 8: e56728. [Crossref]

18. Huston JM, Gallowitsch-Puerta M, Ochani M, Ochani K, Yuan R, et al. (2007) Transcutaneous vagus nerve stimulation reduces serum high mobility group box 1 levels and improves survival in murine sepsis. Crit Care Med 35: 2762-2768. [Crossref]

19. Koopman FA, Koopman FA, Chavan SS, Miljko S, Grazio S, et al. (2016) Vagus nerve stimulation inhibits cytokine production and attenuates disease severity in rheumatoid arthritis. Proc Natl Acad Sci U S A 113:8284-8289. [Crossref]

20. Stavrakis S, Humphrey MB, Scherlag B, Iftikhar O, Parwani P, et al. (2017) LowLevel Vagus Nerve Stimulation Suppresses Post-Operative Atrial Fibrillation and Inflammation: A Randomized Study. JACC Clin Electrophysiol 3: 929-938. [Crossref]

21. Bonaz B, Sinniger V, Hoffmann D, Clarençon D, Mathieu N, Dantzer C, et al. (2016) Chronic vagus nerve stimulation in Crohn's disease: a 6-month follow-up pilot study. Neurogastroenterol Motil 28: 948-953. [Crossref]

22. Liu A, Rong P, Gong L, Song L, Wang X, et al. (2018) Efficacy and Safety of Treatment with Transcutaneous Vagus Nerve Stimulation in 17 Patients with Refractory Epilepsy Evaluated by Electroencephalogram, Seizure Frequency, and Quality of Life. Med Sci Monit 24: 8439-8448. [Crossref]

23. Pavlov VA, Tracey KJ (2012) The vagus nerve and the inflammatory reflex - linking immunity and metabolism. Nat Rev Endocrinol 8: 743-754. [Crossref]

24. van Westerloo DJ, Giebelen IAJ, Florquin S, Daalhuisen J, Bruno MJ, et al. (2005) The cholinergic anti-inflammatory pathway regulates the host response during septic peritonitis. J Infect Dis 91: 2138-2148. [Crossref]

25. Ben-Menachem E (2002) Vagus nerve stimulation for the treatment of epilepsy. Lance Neurol 8: 477-482. Lancet Neurol 1: 477-482. [Crossref]

26. Staats P, Giannakopoulos G, Blake J, Liebler E, Levy RM (2020) Use of non-inveasive vagus nerve stimulation to treat respiratory symptoms associated with COVID-19: A theoretical hypothesis and early clinical experience. Neuromodulation 13172. [Crossref]

Copyright: (C2020 Nemechek P. This is an open-access article distributed under the terms of the Creative Commons Attribution License, which permits unrestricted use, distribution, and reproduction in any medium, provided the original author and source are credited. 